\title{
Sex differences in antibody- and cell-mediated immune response to rubella re-immunisation
}

\author{
LESLIE A. MITCHELL \\ Departments of Pathology and Pediatrics, University of British Columbia, Vancouver, BC V5Z 4H4, Canada
}

\begin{abstract}
Antibody (AMI) and cell-mediated (CMI) immunity to rubella virus (RV) were evaluated in healthy adolescent males $(n=11)$ and females $(n=28)$ undergoing routine reimmunisation with $\mathrm{RA27} / 3$ strain $\mathrm{RV}$ as a component of measles-mumps-rubella (MMR) vaccine. Blood samples were collected just before and at 2,4 and 10 weeks after MMR. While there were no sex differences before MMR and at week 10 after vaccination, levels of specific IgG determined by whole RV enzyme immunoassay were found to be significantly higher in males at weeks 2 and 4, suggesting brisker onset of recall AMI. Analysis of RV protein-specific IgG by immunoblot assays also revealed that while there were no notable sex differences in the distribution of E1-specific antibodies, more males produced E2-specific antibodies whereas more females produced C-specific antibodies after immunisation. Analysis of CMI with whole inactivated RV and a panel of RV synthetic peptides in lymphocyte proliferation assays revealed a brisker onset of CMI in males which paralleled that observed for AMI. The numbers of RV antigens recognised by males were significantly higher at weeks 2 and 4. Also, mean and median stimulation indices measured at weeks 2 and 4 for certain peptides, including two known to contain overlapping antibody neutralisation domains and T-cell epitopes, E1(213-239) and E1(254-285), were also found to be significantly higher in male subjects. These observations suggest that there are hormonal influences on RV-specific immunity which might result in differential handling of $\mathrm{RV}$ and, hence, may partially explain why females are more predisposed to adverse outcomes of rubella infection and immunisation.
\end{abstract}

\section{Introduction}

An earlier study demonstrated sex differences in the kinetics and specificity of the human antibody response to rubella virus (RV) structural proteins $\mathrm{E} 1, \mathrm{E} 2$ and $\mathrm{C}$ [1]. That study examined the IgM, IgA and IgG antibody responses of 32 males and 67 females during acute and convalescent phases following wild-type RV infection. Earlier onset and higher levels of IgG, IgA and $\operatorname{IgM}$ antibodies directed to the $\mathrm{E} 1$ envelope protein known to contain most of the virus neutralisation domains, as well as to a particular E1 peptide, E1(213239 ), containing a linear neutralisation domain [2-4],

Received 26 Jan. 1999; accepted 21 April 1999.

Corresponding author: Dr L. A. Jonsen (Mitchell). Present address: BC Research Institute for Children's and Women's Health, 950 West 28th Avenue, Vancouver, BC V5Z 4H4, Canada (e-mail: leslie@wpog.childhosp.bc.ca). were observed in males. However, convalescent sera from females had higher levels of IgG antibodies directed to whole RV. That study also revealed a higher proportion of E2-specific IgG and IgA antibodies in female sera. These differences were of interest considering the higher incidence of adverse sequelae of rubella, such as joint manifestations, in women [5].

The present study investigated RV-specific antibody levels and cell-mediated immunity (CMI) before and at 2,4 and 10 weeks after vaccination in adolescents undergoing re-immunisation with live attenuated RA27/3 strain RV as a component of trivalent measles-mumps-rubella (MMR) vaccine. Antibody levels were measured by whole RV EIA and RV protein-specific immunoblot. Inactivated whole RV and a panel of synthetic peptides representing selected regions of E1, E2 and $\mathrm{C}$ were used in lymphocyte proliferation assays to determine the specificity and magnitude of the anamnestic response of long-lived RV-specific $T$ cells persisting after earlier MMR immunisation. 


\section{Materials and methods}

Study subjects, immunisation and blood sampling

Study subjects were adolescent males $(n=11$, aged 15.9 SD 0.9 years) and females $(n=28$, aged 15.3 SD 0.5 years), residing in the Vancouver area who were undergoing routine re-immunisation with MMR vaccine to boost immunity to measles. Informed consent from parents and study subjects was obtained as required by the University of British Columbia Research Ethics Committee. Written documentation of previous MMR vaccination was also obtained. A pre-vaccine blood sample was drawn by sterile venepuncture and the subject was immediately vaccinated with MMR (Meruvax II, Connaught Laboratories, Willowdale, Ontario, Canada). Follow-up blood samples were obtained at 2, 4 and 10 weeks after MMR vaccination. Reactions to the vaccine were also assessed at these intervals. Sera were separated and stored at $-20^{\circ} \mathrm{C}$ until testing.

\section{Determination of $R V$-specific antibodies}

Levels of whole RV-specific IgG antibodies were determined by the Rubella Enzygnost EIA (Behringwerk, Marburg, Germany) according to the manufacturer's directions. A negative cut-off of $0.200 \mathrm{ab}-$ sorbance unit (equivalent to $10 \mathrm{IU} / \mathrm{ml}$ ) was used. RV protein-specific IgG was determined by immunoblot assays performed under non-reducing conditions as described previously [6]. A positive result was determined by the appearance of one or more bands at the known positions of the E1, E2 and C proteins on the blot with reference to a pooled serum control containing antibodies of all three specificities.

\section{Rubella virus antigens}

A panel of RV synthetic peptides representing selected regions of RV E1, E2 and C proteins (Table 1) was prepared by automated solid-phase synthesis by the FMOC procedure (Synergy, Applied Biosystems, Mississauga, ON, Canada). Quality checks of peptide products were performed by HPLC. Whole RV (M33 strain), isolated from RV-infected Vero cell culture supernates by ultracentrifugation, was heat-inactivated at $56^{\circ} \mathrm{C}$ for $30 \mathrm{~min}$ and used as a positive control antigen.

\section{Lymphocyte proliferation assays}

Lymphocyte proliferation assays were performed as described previously [7]. At each study interval, peripheral blood mononuclear cells (PBMNC) were isolated from heparinised blood by density gradient centrifugation through Ficoll-Hypaque (Pharmacia, Dorval, Quebec, Canada), washed three times by centrifugation, then added at an initial density of 100000 cells/well in complete OptiMEM (Gibco/ BRL, Mississauga, ON, Canada) containing penicillin 10 units $/ \mathrm{ml}$, streptomycin $10 \mathrm{mg} / \mathrm{L}$ and $2 \times 10^{-5} \mathrm{M} \mathrm{2-}$ mercaptoethanol to 96 -well microculture plates. Each well was supplemented with autologous plasma $10 \%$. Peptides were tested in triplicate at a final concentration of $10 \mu \mathrm{M} /$ well - previously determined to be within optimal range for lymphocyte stimulation. Positive control wells received heat-inactivated whole $\mathrm{RV}$ at a concentration equivalent to $10^{3} \mathrm{pfu} /$ well. Wells containing phytohaemagglutinin (PHA) at a final concentration of $2 \mathrm{mg} / \mathrm{L}$ were also used as a positive control. Negative control wells received medium only. Microculture plates were incubated for 7 days at $37^{\circ} \mathrm{C}$

Table 1. Rubella virus synthetic peptides

\begin{tabular}{lll}
\hline Peptide no. & Protein position & Amino-acid sequence \\
\hline 1 & E1(98-126) & LASYFNPGGSYYKQYHPTACEVEPAFGHS \\
2 & E1(205-247) & EYIMNYTGNQQSRWGLGSPNCHGPDWASPVCQ \\
& RHSPDCSRLVG \\
3 & E1(213-239) & NQQSRWGLGSPNCHGPDWASPVCQRHS \\
4 & E1(234-252) & VCQRHSPDCSRLVGATPER \\
5 & E1(254-266) & RLRLVQDADDPLLR \\
6 & E1(254-285) & RLRLVQDADDPLLRIAPGPGEVWVTPVIGSQA \\
7 & E1(258-277) & VDADDPLLRTAPGPGEVWVT \\
8 & E1(272-285) & GEVWVTPVIGSQAR \\
9 & E1(301-314) & VEMDEWIHAHTTSD \\
10 & E1(389-408) & GLLACSAKCLYYLRGAIAPR \\
11 & E1(462-481) & GLQPRADMAAPPTLPQ \\
12 & E2(1-16) & APPTLPQPPRAHGQHYGHHHHQLPFLG \\
13 & E2(10-36) & LGHDGHHGGTLRVGQHHRNASDVL \\
14 & E2(35-58) & LRVGQHYRNASDVLPGHWLQ \\
15 & E2(45-64) & HYRNASDVLPGHWLQGGWGCYNL \\
16 & E2(50-72) & GFLSGVGPMRLRHGADT \\
17 & E2(134-150) & GPMRLRHGADTRSGRLI \\
18 & E2(140-156) & TRFGCAMRWGLPP \\
19 & E2(168-179) & WVCIFMVCRRACR \\
20 & E2(248-260) & MASTTPITMEDLQKALEAQSRALRAGLAA \\
21 & C(1-29) & RSQTPAPKPSRAPPQQPQPPRMQT \\
22 & C(88-111) & LHDPDTEAPTEACVTSWL \\
23 & C(141-158) & VRAYNQPAGDVRGVWGKGERTYAEQDFRV \\
24 & C(206-234) & TRWHRLLRMPVR \\
25 & C(237-248) &
\end{tabular}


in $\mathrm{CO}_{2} 5 \%$ in air. To measure lymphocyte proliferation, cells were pulsed with ${ }^{3} \mathrm{H}$-thymidine $2 \mu \mathrm{Ci}$ /well during the last $6-8 \mathrm{~h}$ of the culture period. DNA was harvested from each well and incorporated radioactivity was determined. Stimulation indices (SIs) were calculated from the mean cpm determined in stimulated wells divided by the mean cpm determined in negative control wells. SIs $\geqslant 2.0$ indicated a positive cell response to a given antigen.

\section{Data analysis}

Differences in mean and medium antibody titres, SIs for RV or individual peptide antigens, were determined by the Student's $t$ test or Mann-Whitney $U$ test. Confidence intervals $(95 \% \mathrm{CI})$ for the differences in proportion were calculated and differences were considered significant at the $p<0.05$ level if zero was excluded.

\section{Results}

\section{Study subjects}

A total of 39 subjects ( 11 males and 28 females) was recruited for the study. Of these, $22(56.4 \%)$ were white, $11(28.2 \%)$ were Chinese and $6(15.4 \%)$ were Filipino or Eurasian. There were no significant differences in ethnic distribution or age between males and females in the study. All study subjects provided documentation of previous MMR vaccination given between the ages of 12 and 15 months. One study subject of USA origin had received a second dose of MMR vaccine 6 years previously according to the requirements of the state in which he had been a resident. The remaining study subjects received their second dose of MMR as participants in this study. All but one subject completed follow-up to 10 weeks after vaccination. No subjects reported adverse sequelae (paresthesiae, myalgia, joint manifestations, etc.) as a result of MMR revaccination. In the 4 years prior to, and during the course of the study, there was no increase in the incidence of natural rubella disease in the community.

Sex differences in rubella-specific antibody levels before and after MMR re-immunisation

Whole RV-specific IgG levels were determined by EIA in sera collected just before and at 2, 4 and 10 weeks after MMR vaccination (Table 2). Although there were no sex differences in pre-vaccine and week 10 antibody levels, total RV-specific antibody levels were significantly higher in males than in females at weeks 2 and 4. IgG antibodies directed to RV structural proteins including envelope glycoproteins (E1 and E2) and the internal capsid (C) protein were also determined at these study intervals by non-reducing immunoblot assays. Before vaccination, all but one subject (female) had antibodies directed to E1; after MMR, all subjects

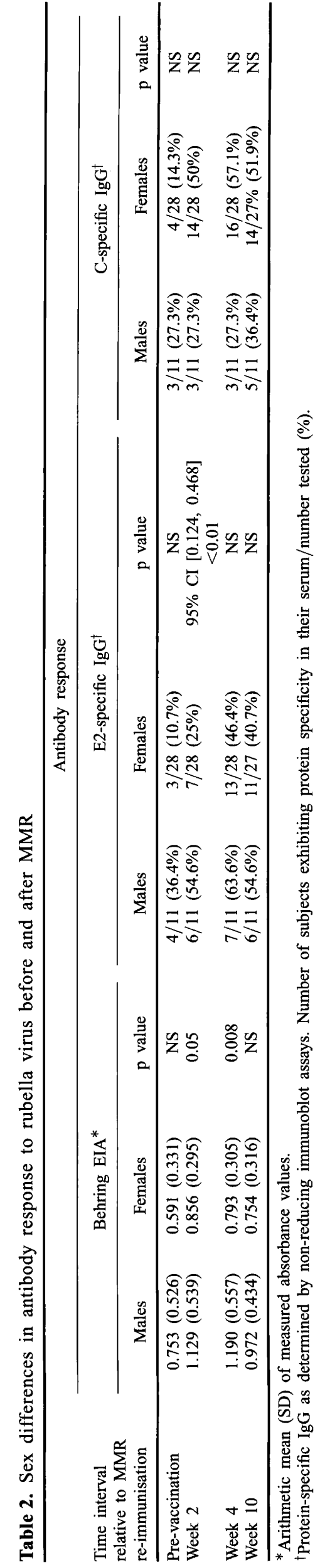


had E1-specific antibodies (data not shown). The proportion of males $(54.6 \%)$ with E2-specific antibodies at week 2 was higher $\mathrm{p}<0.01$ ) than the proportion of females (Table 2). However, significant differences were not observed for the other study intervals or for the proportions of male and female subjects with $\mathrm{C}$-specific antibodies, although the proportions of females expressing $\mathrm{C}$-specific antibodies were found to be higher at all post-vaccine study intervals (Table 2). In an earlier study of rubella reimmunisation [8], an increase in the number of RV protein specificities (in particular, the appearance of Cspecific IgG) expressed in study subjects who lacked antibody directed to a linear neutralisation domain represented by the cyclised peptide, $\mathrm{BCH}-178 \mathrm{c}-$ E1(213-239), but who were otherwise RV seropositive (as determined by whole virus EIA) was observed. It was hypothesised that the lack of antibody directed to this neutralisation domain may have allowed the vaccine strain virus to replicate and therefore to stimulate antibody species directed to the $C$ protein. Study subjects who had E1 peptide-specific antibody levels $>10 \mathrm{IU} / \mathrm{ml}$ before immunisation did not show this type of response. Although it was not possible to measure antibodies directed to this peptide in the present study, due to inavailability of $\mathrm{BCH}-178 \mathrm{c}$, it was observed that $21(75 \%)$ of 28 female subjects in comparison with $4(36.4 \%)$ of 11 male subjects $(\mathrm{p}<0.01 ; 95 \% \mathrm{CI}=0.057,0.715)$ demonstrated an increase in complexity of the immunoblot pattern (i.e., the appearance of C-specific antibodies and in all except five subjects, antibodies directed to E2 as well).

\section{Sex differences in CMI to rubella antigens before and after MMR vaccination}

CMI to RV (heat-inactivated M33 strain) and to RV peptides (Table 1) was assessed by lymphocyte proliferation assays in samples collected just before and at 2, 4 and 10 weeks after MMR. Peptides shown in Table 1 were recognised to varying degrees by all study subjects [9]. At weeks 2 and 4 both the mean and median numbers of peptides recognised by male subjects were significantly higher than in female subjects (Table 3). In examining arithmetic mean SIs for individual peptides (Table 4), significantly higher values were observed at weeks 2 and 4 after MMR in males than in females for the following antigens: whole RV, E1(213-239), E1(254-285), E1(389-408), E2(4564), E2(134-150) and $C(237-248)$. At week 10 significantly higher mean SIs were observed in females in response to the following peptides: E1(301-314), E2(134-150), E2(168-179) and E2(248-260). As the range of values observed in lymphocyte proliferation assays is inherently large, the study also compared sex differences in median SIs. At week 2 after stimulations

Table 3. Sex differences in cell-mediated immunity to RV

\begin{tabular}{lccc}
\hline $\begin{array}{l}\text { Time interval } \\
\text { relative to MMR } \\
\text { re-immunisation }\end{array}$ & Males & Females & p-value \\
\cline { 2 - 4 } & $5.2(3.0)$ & $5.4(3.0)$ & NS (NS) \\
Pre-vaccination & $8.8(9.0)$ & $5.1(5.0)$ & $0.017(0.0371)$ \\
Week 2 & $7.2(7.0)$ & $4.2(3.0)$ & $0.002(0.0038)$ \\
Week 4 & $9.3(9.0)$ & $11.0(12.0)$ & NS (NS) \\
Week 10 & & \\
NS, not significant & & \\
* Arithmetic mean number (median) of RV peptide antigens recognised (SI $\geqslant 2.0)$ in the test \\
panel (20-23 peptides tested/study interval). Sex differences in mean were determined by \\
Student's test, while differences in median values were determined by Mann-Whitney U tests.
\end{tabular}

Table 4. Kinetic differences between males and females in lymphocyte recognition of RV antigens after re-immunisation

\begin{tabular}{lccc}
\hline & \multicolumn{2}{c}{ Mean (median) stimulation index } & Significance \\
\cline { 2 - 3 } Interval/peptide & Males $(\mathrm{n}=11)$ & Females $(\mathrm{n}=28)$ & \begin{tabular}{c}
$\mathrm{p}$ value) \\
\hline Week 2
\end{tabular} \\
E1(213-239) & $3.7(1.2)$ & $1.0(0.7)$ & 0.047 \\
E1(389-408) & $7.4(4.3)$ & $1.8(0.8)$ & 0.003 \\
Week 4 & & & \\
Whole RV & $18.4(8.1)$ & $7.9(2.0)$ & 0.006 \\
E1(254-285) & $3.3(0.9)$ & $1.2(0.8)$ & 0.015 \\
E1(389-408) & $2.1(1.6)$ & $1.0(0.9)$ & 0.006 \\
E2(45-64) & $2.8(2.3)$ & $1.8(1.7)$ & 0.034 \\
E2(134-150) & $2.3(1.9)$ & $1.5(1.4)$ & 0.026 \\
C(237-248) & $5.4(5.3)$ & $2.7(2.2)$ & 0.011 \\
Week 10 & & & \\
E1(301-314) & $1.1(0.8)$ & $4.2(1.7)$ & 0.004 \\
E2(134-150) & $1.6(1.2)$ & $9.8(4.2)$ & 0.004 \\
E2(168-179) & $1.2(1.1)$ & $6.3(1.4)$ & 0.028 \\
E2(248-260) & $3.6(2.6)$ & $10.3(2.5)$ & 0.021 \\
\hline
\end{tabular}


with E1(389-408), median SIs for the male group (4.3) were significantly higher $(p=0.0026)$ than for female subjects (0.8). Similarly, at week 4 median SIs for whole RV $(p=0.0057)$ and the capsid peptide $C(237-$ 248) $(p=0.0107)$ were higher in the male group. At week 10 , median SIs for females (4.2) were significantly higher $(p=0.0087)$ than for males $(1.2)$ for peptide E2(134-150).

\section{Discussion}

In an earlier study of immunity to rubella virus following natural infection [1], more rapid onset of, as well as a greater proportion of, IgG antibodies directed to E1, in comparison with E2- and C-specific antibodies was observed in males relative to females. In particular, in the very early acute response (0-6 days after onset of rash) males made higher levels of IgM and IgG directed to a known neutralisation domain represented by a cyclised version (BCH-178c) of the peptide E1(213-239) than did females, although by late convalescence $(0.5-3$ years after onset) mean levels of E1 peptide-specific IgG were higher in females. These observations suggested a potential for more expedient handling of RV in terms of antibodymediated immunity in males. Also, total levels of RVspecific IgG and IgA observed in convalescent serum of females were higher, suggesting that higher levels of RV viraemia or antigenaemia, or both, may have occurred, or possibly longer persistence of viral antigen, leading to enhanced immunostimulation.

The present study examined sex differences in both the antibody and cell response to re-immunisation with live attenuated RA27/3 strain vaccine given as a component of the trivalent MMR vaccine. Subjects were adolescents who had been immunised 12-15 years earlier with MMR. Although there were no sex differences in total whole RV-specific IgG levels before and at week 10 after MMR, levels at weeks 2 and 4 were significantly higher in males than in females. In this study, no difference was observed in the proportion of males and females with E1-specific IgG at any study interval. However, as the relative proportion of the antibody response directed to the individual $\mathrm{RV}$ structural proteins and to the E1 neutralisation domain peptide (BCH-178c) were not evaluated as in the previous study, it is not known if the recall E1-specific antibody response may have been greater in males. In contrast to the study of the antibody response to wildtype virus, higher levels of E2-specific IgG were observed in male sera at week 2 , suggesting a brisker recall response to this vaccine antigen. The higher proportion of males with E2-specific IgG at week 2 is of interest, because of the reported existence of a neutralisation domain on this envelope protein [10]. Also, no gender difference in the $\mathrm{C}$-specific response to the live attenuated vaccine strain RV was observed here in comparison with the infection study in which higher levels were observed in females at 1-4 weeks after onset of rash. While the sex differential in the proteinspecific IgG response to vaccine strain RV differs somewhat from that observed earlier for wild-type infections [1], the results of the present study suggest more rapid onset of the recall response to $\mathrm{RV}$ in males than in females. Thus, the same trend prevails.

Also, notable sex differences in the kinetics of the CMI response to re-immunisation with vaccine strain $\mathrm{RV}$ were observed in that the mean (and median) numbers of peptides in the test panel that were recognised by specimens from male subjects were significantly higher at weeks 2 and 4 than in female subjects, paralleling the kinetic differences observed for the total RVspecific IgG response. Hence, CMI as determined by lymphocyte proliferation response to $\mathrm{RV}$ and $\mathrm{RV}$ peptide antigens, including E1(213-239) and E1 (254-285) known to contain antibody neutralisation domains $[2-4,11]$ as well as immunodominant T-cell epitopes [12,13], also appeared to be brisker in males. Furthermore, significantly higher SIs were observed in male samples at weeks 2 and 4 , for stimulations with peptides E1(389-408) and E2(45-64) which are adjacent to the proposed antibody neutralisation domain on E2 [10]. However, by week 10 SIs for E1 (301-314) and E2(134-150) were higher in female specimens. The significance of this recognition is not known as, so far, no biological functions have been ascribed to these regions of E1 and E2.

Thus, the results of this study reveal kinetic differences between males and females in the recall response of both $\mathrm{B}$ and $\mathrm{T}$ lymphocytes to rubella antigens, which suggest hormonal influences on the immune response to RV. Whether this translates into more expedient functional immunity (i.e., generation of neutralising antibodies or cytotoxic $\mathrm{T}$ cells) requires further study. However, if so, these kinetic differences may partly explain why adverse outcomes of RV infection and vaccination (such as joint manifestations) are rarely seen in males or prepubertal children of either sex [5]. Insofar as persistent RV infection has been suggested as a possible mechanism $[14,15]$, a slower onset of RV-specific cell-mediated and antibody-mediated immunity in females may allow for increased viraemia with escape of the virus to atypical tissue sites such as the synovial space. No adverse reactions to RV immunisation were observed in the present study. This was not surprising given the small number of subjects studied and their ages, as the incidence of rubella vaccine-related acute joint manifestations in adult females is in the order of $10 \%$ [16] and is related to increasing age [17]. However, occurrence of acute and chronic arthritis following secondary rubella immunisation in children has been observed [18, and A. J. Tingle, unpublished observations]. While the present study results suggest hormonal influences on the RV-specific immune response, the potential influences of other parameters 
such as immunogenetic background on recognition of RV antigens cannot be excluded.

This study was supported by funds from the British Columbia Children's Hospital Telethon Clinical Investigation Initiatives (no. 25207). I gratefully acknowledge Dr Maan Zrein (formerly of BioChem Immunodiagnostics) for advice on rubella peptide design, Patti Janssen for help with statistical analyses and Dr Aubrey J. Tingle (B. C. Research Institute for Children's and Women's Health) for helpful discussion

\section{References}

1. Mitchell LA, Zhang T, Tingle AJ. Differential antibody responses to rubella virus infection in males and females. $J$ Infect Dis 1992; 166: 1258-1265.

2. Mitchell LA, Zhang T, Ho $\mathrm{M}$ et al. Characterization of rubella virus-specific antibody responses by using a new synthetic peptide-based enzyme-linked immunosorbent assay. $J$ Clin Microbiol 1992; 30: 1841-1847.

3. Chaye H, Ou D, Chong P, Gillam S. Human T- and B-cell epitopes of El glycoprotein of rubella virus. J Clin Immunol 1993; 13: 93-100.

4. Wolinsky JS, Sukholutsky E, Moore WT, Lovett A, McCarthy M, Adame B. An antibody- and synthetic peptide-defined rubella virus E1 glycoprotein neutralization domain. $J$ Virol 1993; 67: 961-968

5. Howson CP, Howe CJ, Fineberg HV. Adverse effects of pertussis and rubella vaccines. Washington, DC, National Academy Press. 1991: 1-8.

6. Zhang T, Mauracher CA, Mitchell LA, Tingle AJ. Detection of rubella virus-specific immunoglobulin $\mathrm{G}(\mathrm{IgG}), \operatorname{IgM}$, and $\operatorname{IgA}$ antibodies by immunoblot assays. J Clin Microbiol 1992; 30: 824-830.

7. Mitchell LA, Décarie D, Tingle AJ, Zrein M, Lacroix $M$. Identification of immunoreactive regions of rubella virus $\mathrm{E} 1$ and E2 envelope proteins by using synthetic peptides. Virus Res 1993; 29: 33-57.

8. Mitchell LA, Ho MK-L, Rogers JE et al. Rubella reimmunization: comparative analysis of the immunoglobulin $G$ response to rubella virus vaccine in previously seronegative and seropositive individuals. $J$ Clin Microbiol 1996; 34: 2210-2218.

9. Mitchell LA, Tingle AJ, Decarie D, Shukin R. Identification of rubella virus $T$-cell epitopes recognized in anamnestic response to RA27/3 vaccine: associations with boost in neutralizing antibody titer. Vaccine 1999 (in press).

10. Green KY, Dorsett PH. Rubella virus antigens: localization of epitopes involved in hemagglutination and neutralization by using monoclonal antibodies. $J$ Virol 1986; 57: 893-898.

11. Terry GM, Ho-Terry L, Londesborough P, Rees RK. Localization of the rubella E1 epitopes. Arch Virol 1988; 98: 189-197.

12. Ou D, Mitchell LA, Ho M et al. Analysis of overlapping Tand B-cell antigenic sites on rubella virus E1 envelope protein. Influence of HLA-DR4 polymorphism on T-cell clonal recognition. Hum Immunol 1994; 39: 177-187.

13. Edson SE, Lovett A, Sukholutsky E, Wolinsky JS. A synthetic chimeric peptide rapidly induces rubella virus-specific neutralizing antibodies (Abstract). American Society for Virology Meeting, Davis, CA, July 10-14, 1993.

14. Tingle AJ, Pot KH, Chantler JK. Prolonged arthritis, viraemia, hypogammaglobulinemia, and failed seroconversion following rubella immunisation. Lancet $1984 ; 1$ : $1475-1476$.

15. Chantler JK, Ford DK, Tingle AJ. Persistent rubella infection and rubella-associated arthritis. Lancet 1982; 1: 1323-1325.

16. Tingle AJ, Mitchell LA, Grace $M$ et al. Randomised doubleblind placebo-controlled study on adverse effects of rubella immunisation in seronegative women. Lancet 1997; 349: 1277-1281.

17. Weibel RE, Stokes J, Buynak EB, Hilleman MR. Influence of age on clinical response to HPV-77 duck rubella vaccine. JAMA 1972; 222: 805-807.

18. Jacobs JC. Childhood arthritis following rubella booster immunizations: is arthritis more frequent in immune individuals? Pediatr Res 1991; 29: 175A (abstract). 